\title{
Treatment of Rectal Cancer with the Paleolithic Ketogenic Diet: A 24-months Follow-up
}

\author{
Csaba Tóth, Zsófia Clemens* \\ Paleomedicina Hungary Ltd, Evolutionary Medicine Working Group, Budapest, Budapest, Hungary \\ *Corresponding author: clemenszsofia@gmail.com
}

\begin{abstract}
Background: Ketogenic diets have repeatedly been suggested for the treatment of cancer. Yet, only a few case studies have been published that reported long term benefits associated with such diets. Case report: Here we present a case where rectal cancer was treated with an animal fat-meat based diet, which we refer to as the paleolithic ketogenic diet. Upon diet initiation, the patient discontinued three medicines he had been taking because of hypertension and hyperuricemia. The patient initially received 6 weeks of radiation therapy. Thereafter the patient used the diet as a stand-alone therapy for 22.5 months. The patient was not taking any medicines or dietary supplements while on the diet. During the first five month of the diet therapy the patient exhibited excellent adherence which was paralleled by improving laboratory parameters including decreasing tumor marker levels and decreasing tumor size. The patient was in a constant ketosis during the entire follow-up. From the seventh month on, however, his adherence level was fluctuating with periods of worse adherence paralleled with negative changes in laboratory parameters. Although MRI imaging showed that there was no increase in the size of the tumor, 22 months after diagnosis onset the patient reported an alteration in bowel habits and that the bloody stool had returned. Because of the emergence of these symptoms, 24 months after diagnosis onset, rectal surgery was performed. Conclusion: With using the paleolithic ketogenic diet the patient was able to postpone surgery for two years. During the first five months, when the patient was strictly adhering to the diet, the tumor regressed. Thereafter, with incomplete dietary adherence, disease seemed to be stable but symptoms suggested progression for the last few months of the follow-up. Deviations from diet rules, even those not affecting ketosis, resulted in the progression of the disease.
\end{abstract}

Keywords: rectal tumor, colorectal cancer, paleolithic diet, ketogenic diet, paleolithic ketogenic diet, metabolic therapy

Cite This Article: Csaba Tóth, and Zsófia Clemens, "Treatment of Rectal Cancer with the Paleolithic Ketogenic Diet: A 24-months Follow-up.” American Journal of Medical Case Reports, vol. 5, no. 8 (2017): 205-216. doi: 10.12691/ajmcr-5-8-3.

\section{Introduction}

Ketogenic diets have been shown to confer benefits in several pathological conditions including systemic $[1,2]$ as well as neurologic [3] disorders. Carbohydrate-restricted diets have also been suggested as a promising therapeutic approach in tumors $[4,5,6]$. However, until now no more than a few papers reported on the use of the ketogenic diet in cancer patients. Case studies with a positive effect include a landmark paper from 1995 [7] reporting longterm survival of two children diagnosed with malignant brain cancer. Another case report indicated halted progression of glioblastoma multiforme while on the ketogenic diet [8]. Two group studies [9,10] reported stable disease in those cancer patients with sustained ketosis, however both studies were limited in duration with three and one month follow-up, respectively. In a recent study six cancer patients used the ketogenic diet concurrently with radiotherapy or radiochemotherapy [11] which resulted in tumor regression in those with early stage disease. This study was, however, also limited in follow-up duration, so the contribution of diet versus the radiation treatment cannot be discerned. In a study with patients with recurrent glioblastoma no significant clinical effect was seen [12] while in a retrospective study with brain cancer patients, survival benefit was uncertain [13].

Benefits of the ketogenic diet are often explained evolutionarily [14]. An increasing number of studies, as also reviewed by a recent metaanalysis [15], indicate metabolic benefits of the human evolutionary diet also known as the stone age diet [16] or paleolithic diet [17]. In recent years we have published a series of case studies where type 1 diabetes [18,19], type 2 diabetes [20], epilepsy [21,22], Gilbert's syndrome [23] and Crohn's disease [24] where was successfully treated by the paleolithic ketogenic diet, an animal fat-meat based diet. In our most recent case study we reported on halted progression of soft palate cancer for 20 months in a patient on the paleolithic ketogenic diet [25]. The paleolithic ketogenic diet that we use, differs from both the ketogenic diet (as described in the classical ketogenic literature) and 
the paleolithic diet (as described in recent clinical papers using this term).

Here we present a case of a patient with rectal tumor. The patient was started on the paleolithic ketogenic diet shortly after diagnosis onset. Initially he also received concurrent radiotherapy for six weeks. Later on, the diet was used as a stand-alone therapy for 22.5 months.

\section{Case Report}

\subsection{Previous Medical History}

Previous medical history of the 62-year-old patient included hypertonia since the age of 30 controlled with antihypertensives for three decades. For the two years before the diagnosis of rectal tumor, the patient reported an intermittent presence of bloody stool. For the two months before diagnosis onset, he reported frequent diarrhea.

On 18 May 2015 a CT examination of the abdomen and pelvis was carried out which showed a 6-cm long thickening of the rectal wall. On 19 May 2015 colonoscopy confirmed the neoplasm. Biopsy was taken and histopathology showed adenocarcinoma intestini crassi. Additional examinations, including chest radiograph indicated no distant metastasis. The patient was staged as T3N1M0. The patient was offered radiochemotherapy as a neoadjuvant therapy before surgery. The patient accepted radiotherapy but refused chemotherapy. Upon completing the radiotherapy the patient decided not to have surgery.

\subsection{Paleolithic Ketogenic Diet}

We met the patient shortly after his diagnosis and advised the paleolithic ketogenic diet which the patient initiated on 28 May 2015. The paleolithic ketogenic diet is a meat-fat based diet similar to that originally proposed by Voegtlin [16] with a fat:protein ratio of about 2:1 (in grams). Fat and red meats over lean meats were encouraged as well as a regular intake of organ meats. Cereal grains, milk and dairy, vegetable oils (including coconut oil and olive oil), oilseeds, nightshades, legumes, refined sugars, artificial sweeteners and foods with additives were excluded. Vegetables, mostly in the form of root vegetables, were allowed to an extent of $<30 \%$ (in volume) of the diet. It was also suggested to decrease vegetables as much as possible. Fruits and alcoholic drinks were not allowed. Small amount of honey was allowed for sweetening. Coffee was allowed in moderation. We advised against taking vitamin, mineral and any other supplements and/or medicines so the patient was not taking any of these. At the time of writing the manuscript the patient is on the paleolithic ketogenic diet for 24 months without interruption.

When we first met the patient, he was taking atenolol and rilmenidine for hypertension, and allopurinol because of hyperuricemia. Upon diet onset, the patient was suggested to discontinue allopurinol and rilmenidine promptly. Atenolol was discontinued gradually within two weeks.

The patient was under our close control with frequent several personal visits, e-mail and phone communications.

\subsection{Adherence}

Adherence was assessed by regular laboratory workups, daily home monitoring of weight, blood pressure, pulse, blood glucose and urinary ketones as well as regular patient feedback. Urinary ketosis was measured on a daily basis using ketone strips for semi-quantitative measurement of ketones. Notably, urinary ketones were positive on each day of the follow-up during the 24 months (Figure 1). Laboratory ketones were also positive on each measurement and glucose levels were low (Table 1 and Table 2) indicative of sustained ketosis. However, after November 2015 (thus after five months of high level of dietary adherence), there were a few parameters, including elevated blood pressure and mild iron deficiency anaemia which indicated loosening of dietary adherence. The patient admitted having consuming alcoholic drinks in moderation and an increase in his coffee consumption. The patient reported that it was easy for him to maintain ketosis but found it extremely difficult to maintain all limitations of the paleolithic ketogenic diet in the long term. From this time on the patient was unable to exclude all non-paleolithic ketogenic food items for longer time periods despite several warnings from our side. His food diary indicated the consumption of alcoholic drinks with variable frequency, an increased coffee intake, as well as occasional consumption of tomato and oilseeds. He continued refraining from cereals, milk, dairy, vegetable oils, legumes, nightshades and refined sugars. Despite the lapses in the paleolithic ketogenic diet, he remained in stable ketosis possibly because of the low quantity of the non-paleolithic ketogenic food items. His 17th follow-up laboratory exam (on 31 Jan 2017) indicated large increases in inflammatory markers (CRP: $97.4 \mathrm{mg} / \mathrm{l}$; ESR: $60 \mathrm{~mm} / \mathrm{h}$; fibrinogen: $6.7 \mathrm{~g} / \mathrm{l})$. At this time, he also experienced multiple diarrhea a day. This time we strongly advised the patient to return to the strictest form of the diet, a full meat-fat diet for three weeks. The patient realized this. The next laboratory exam which was performed at the end of the three-week full meat-fat period (on 23 Feb 2017) indicated normalization of all inflammatory markers (CRP: 2,5 mg/l; ESR: $10 \mathrm{~mm} / \mathrm{h}$, fibrinogen: $4 \mathrm{~g} / \mathrm{l})$. Along with this the patient reported cessation of diarrhea. However, thereafter the patient was again unable to maintain all restrictions of the paleolithic ketogenic diet.

\subsection{Radiotherapy}

Radiation therapy was performed for a period of six weeks, shortly after diet initiation, between 12 Jun and 29 July 2015 with daily doses of 1.8 Gy resulting in a total 50.4 Gy dose. The patient declined concurrent chemotherapy. Following the completion of radiation therapy, the patient refused other conventional treatment options, including additional radiotherapy sessions as well as surgery, and used the dietary therapy as a stand-alone therapy for the next 22.5 months. Given the unconventional nature of the treatment the patient had a close follow-up using imaging and laboratory workups.

\subsection{Home monitoring of parameters}

The patient tracked his weight, blood pressure, blood glucose, pulse (Figure 2) and urinary ketones (Figure 1) 
on a daily basis. At diet onset, the patient was moderately overweight (weight: 78kg, $175 \mathrm{~cm}, \mathrm{BMI}=25.5$ ). Following the onset of the paleolithic ketogenic diet he began losing weight (Figure 2A). Currently he weights $65 \mathrm{~kg}$ $(\mathrm{BMI}=21.2)$. Systolic and diastolic blood pressure as well as pulse remained relatively low. From Nov 2015 on, there was a moderate elevation in weight and systolic blood pressure, signifying the beginning of the period of incomplete dietary adherence (Figure 2.B). Blood glucose first showed a decreasing tendency then remained relatively stable and low (Figure 2.C). On average glucose level was $4.7( \pm 0.32) \mathrm{mmol} / \mathrm{l}$. Pulse was relatively stable and low (mean \pm SD: $56.8 \pm 3.9$ beat $/ \mathrm{min}$; Figure 2D).

\section{Urinary ketones}

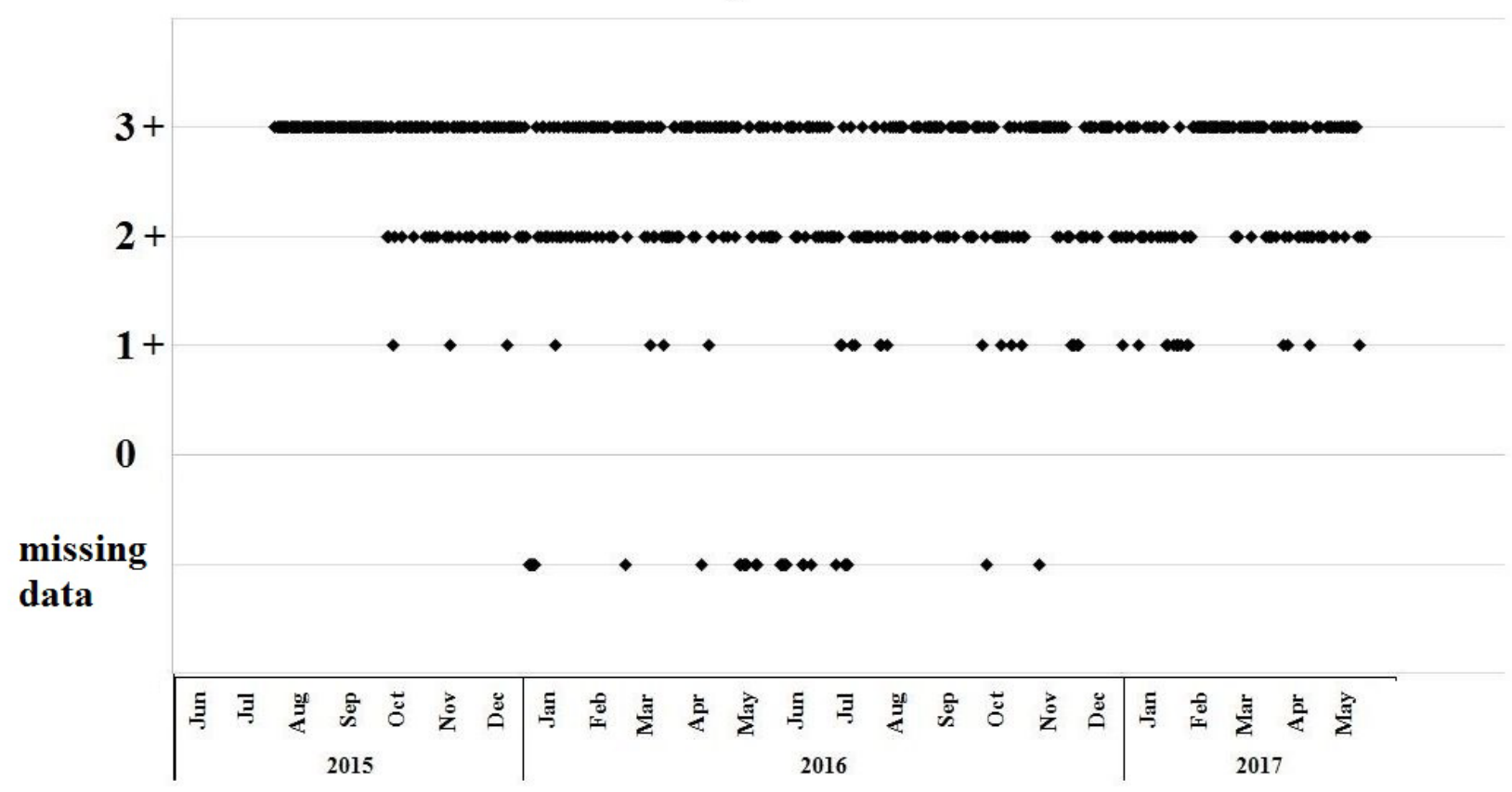

Figure 1. Daily home monitoring of urinary ketosis using urinary ketone strips. 1+: $1.5 \mathrm{mmol} / \mathrm{l}, 2+: 5 \mathrm{mmol} / \mathrm{l}, 3+: 15 \mathrm{mmol} /$. For the first two months of the diet therapy (Jun and July 2015) each measurement indicated ketosis, however scaling was not recorded
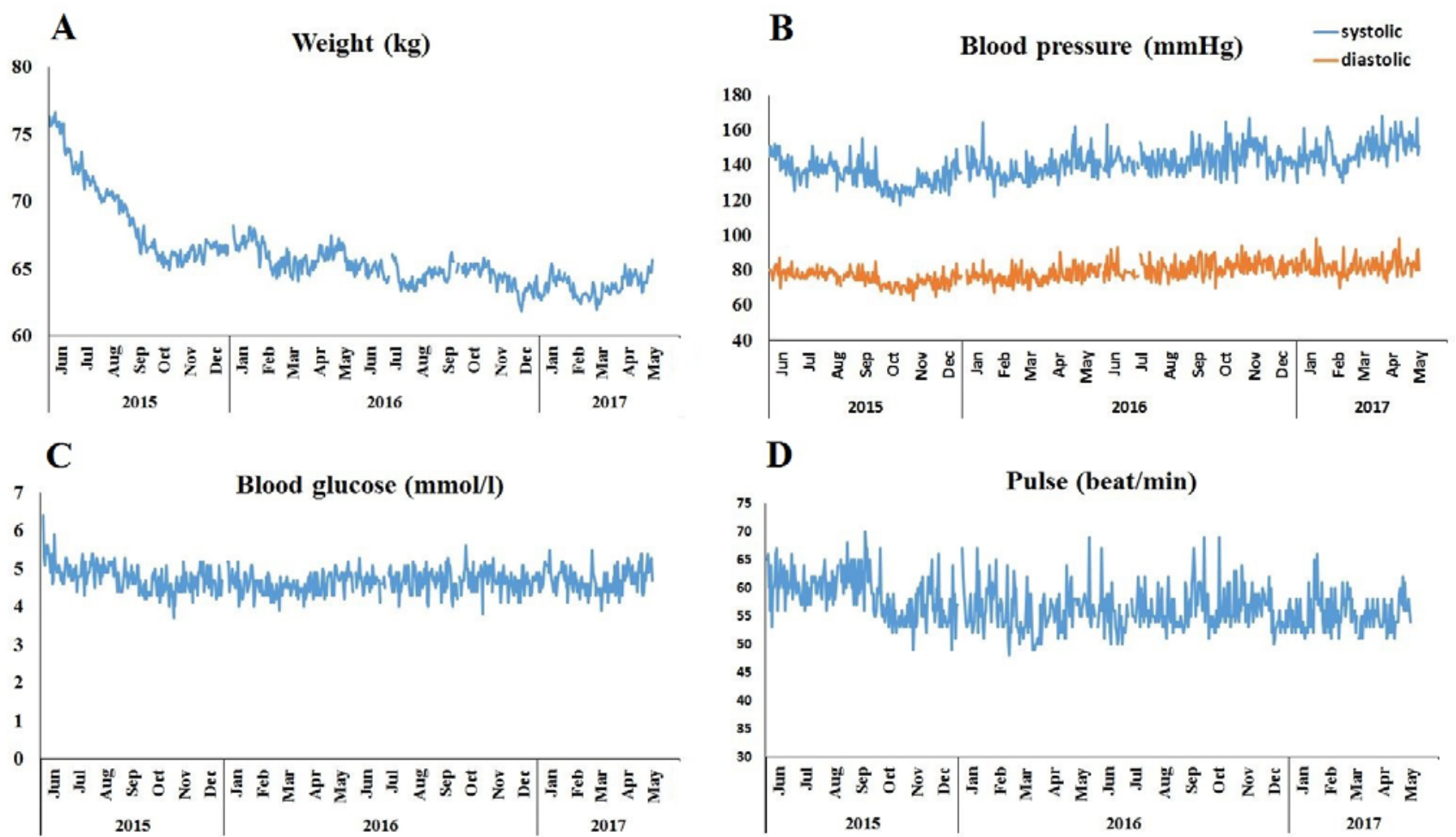

D

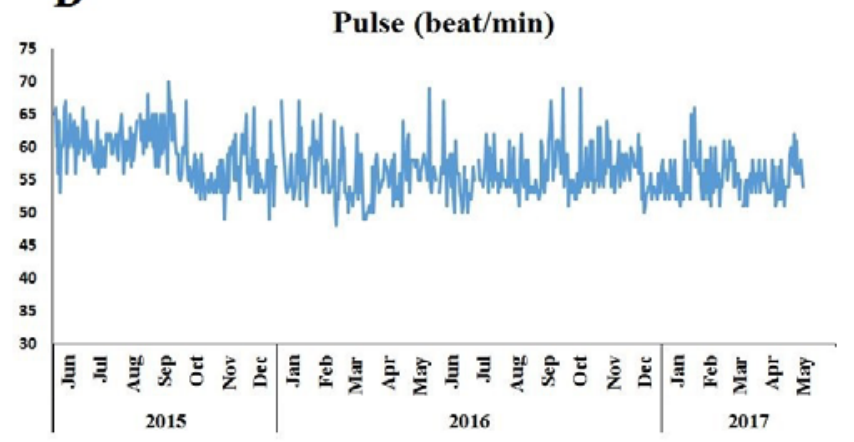

Figure 2. Home monitoring of weight (A), systolic as well as diastolic blood pressure (B), blood glucose (C) and pulse (D) while on the paleolithic ketogenic diet 
Table 1.

\begin{tabular}{|c|c|c|c|c|c|c|c|c|c|c|c|c|c|c|}
\hline & \multicolumn{6}{|c|}{ Standard diet } & \multicolumn{7}{|c|}{ Paleolithic ketogenic diet } & \multirow[b]{3}{*}{ 节 } \\
\hline & & 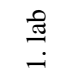 & $\frac{\overrightarrow{\widetilde{\tau}}}{\mathrm{i}}$ & $\begin{array}{l}\text { त्ञी } \\
\dot{m}\end{array}$ & 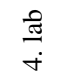 & $\begin{array}{l}\text { त्త్ } \\
\dot{\text { in }}\end{array}$ & $\frac{\overrightarrow{0}}{\dot{0}}$ & $\frac{\pi}{\pi}$ & $\begin{array}{l}\text { त्త్ } \\
\infty\end{array}$ & $\frac{\text { त्తి }}{\sigma}$ & $\begin{array}{l}\frac{\widetilde{\pi}}{0} \\
0\end{array}$ & $\begin{array}{l}\text { శี } \\
\stackrel{-}{=}\end{array}$ & 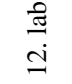 & \\
\hline & 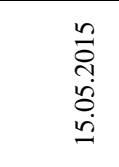 & 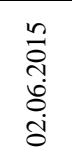 & 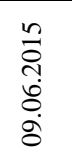 & 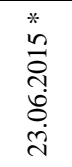 & 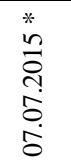 & 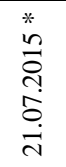 & 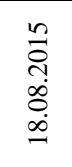 & 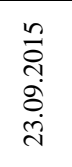 & 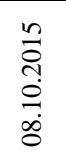 & 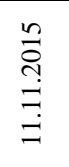 & 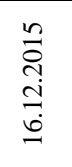 & $\begin{array}{l}0 \\
\stackrel{0}{0} \\
\stackrel{1}{0} \\
0 \\
0 \\
0\end{array}$ & 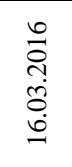 & \\
\hline Glucose & 6 & 5.7 & 5.6 & 5 & 4.8 & 4.3 & 5 & 5.4 & 4.7 & 4.8 & 5 & 5 & 5.8 & $\mathrm{mmo} / \mathrm{l}$ \\
\hline HgA1c & NA & NA & 5.1 & 4.9 & 5.1 & 5.3 & 5.2 & 4.9 & 4.9 & 4.8 & 5.0 & 5.2 & 5.0 & $\%$ \\
\hline Sodium & 144 & 143 & 145 & 142 & 144 & 146 & 143 & 147 & 142 & 144 & 141 & 141 & 143 & $\mathrm{mmol} / \mathrm{l}$ \\
\hline Potassium & 4.5 & 4.0 & 4.7 & 3.9 & 4.2 & 4.9 & 5.2 & 5.0 & 5.2 & 4.5 & 4.5 & 4.8 & 5.2 & $\mathrm{mmol} / \mathrm{l}$ \\
\hline Calcium & NA & 2.6 & 2.57 & 2.41 & 2.39 & 2.48 & 2.37 & 2.59 & 2.54 & 2.43 & 2.48 & 2.49 & 2.46 & $\mathrm{mmol} / \mathrm{l}$ \\
\hline Magnesium & NA & 0.81 & 0.8 & 0.80 & 0.79 & 0.80 & 0.80 & 0.73 & 0.74 & 0.73 & 0.87 & 0.77 & 0.84 & $\mathrm{mmol} / \mathrm{l}$ \\
\hline Carbamide & 5.3 & 6.4 & 5.3 & 3.2 & 4.1 & 5.1 & 4.4 & 6.0 & 4.9 & 5.7 & 6.6 & 5.4 & 6.8 & $\mathrm{mmol} / \mathrm{l}$ \\
\hline Creatinine & 61 & 60 & 61 & 64 & 61 & 64 & 57 & 56 & 57 & 55 & 54 & 62 & 54 & $\mu \mathrm{mol} / \mathrm{l}$ \\
\hline Uric acid & 293 & 608 & 649 & 606 & 409 & 339 & 431 & 373 & 477 & 384 & 307 & 408 & 297 & $\mu \mathrm{mol} / \mathrm{l}$ \\
\hline Total protein & 68 & 75 & 72 & 70 & 65 & 66 & 64 & 67 & 68 & 66 & 68 & 65 & 69 & g/l \\
\hline CRP & 6.1 & 6.4 & 5.8 & 2.6 & 3.5 & 2.6 & 2.2 & 5.5 & 1.0 & 2.5 & 1.1 & 2.6 & 2.0 & $\mathrm{mg} / \mathrm{l}$ \\
\hline HDL & 1.30 & 1.01 & 1.11 & 1.34 & 1.26 & 1.26 & 1.20 & 1.35 & 1.61 & 1.67 & 1.68 & 1.57 & 1.86 & $\mathrm{mmol} / \mathrm{l}$ \\
\hline LDL & 4.75 & 4.89 & 4.49 & 4.08 & 4.02 & 4.29 & 3.62 & 3.68 & 3.95 & 4.17 & 3.80 & 3.62 & 3.82 & $\mathrm{mmol} / \mathrm{l}$ \\
\hline T. cholesterol & 6.8 & 6.2 & 6.2 & 5.5 & 5.4 & 5.8 & 5.1 & 5.3 & 5.8 & 6.0 & 5.7 & 5.5 & 6.1 & $\mathrm{mmol} / \mathrm{l}$ \\
\hline Triglyceride & 0.91 & 0.92 & 0.96 & 0.96 & 0.86 & 0.90 & 0.86 & 0.83 & 0.86 & 0.74 & 0.65 & 0.76 & 0.70 & $\mathrm{mmol} / \mathrm{l}$ \\
\hline GOT & 20 & 21 & 24 & 18 & 13 & 16 & 20 & 16 & 18 & 20 & 20 & 18 & 20 & $\mathrm{U} / \mathrm{l}$ \\
\hline GPT & 20 & 17 & 17 & 16 & 10 & 12 & 16 & 11 & 13 & 15 & 16 & 13 & 13 & $\mathrm{U} / \mathrm{l}$ \\
\hline GGT & 99 & 49 & 38 & 26 & 19 & 20 & 18 & 17 & 16 & 18 & 16 & 18 & 18 & $\mathrm{U} / \mathrm{l}$ \\
\hline ALP & 166 & 62 & 59 & 56 & 53 & 60 & 75 & 92 & 79 & 90 & 96 & 80 & 96 & $\mathrm{U} / \mathrm{l}$ \\
\hline LDH & 256 & 262 & 258 & 276 & 253 & 287 & 310 & 263 & 248 & 272 & 294 & 245 & 266 & $\mathrm{U} / \mathrm{l}$ \\
\hline Amylase & 56 & 57 & 61 & 60 & 67 & 61 & 62 & 62 & 65 & 70 & 68 & 64 & 77 & $\mathrm{U} / \mathrm{l}$ \\
\hline Lipase & NA & 92 & 92 & 91 & 86 & 81 & 65 & 72 & 70 & 84 & 77 & 67 & 72 & $\mathrm{U} / \mathrm{l}$ \\
\hline Iron & NA & 5.6 & 11.4 & 7.7 & 10.4 & 11.9 & 11.9 & 12.8 & 12.7 & 11.3 & 11.7 & 13.8 & 14.2 & $\mu \mathrm{mol} / 1$ \\
\hline Fibrinogen & NA & 5.11 & 4.67 & 4.33 & 3.94 & 3.73 & 4.10 & 4.60 & 3.90 & 3.80 & 3.80 & 3.70 & 4.50 & $g / l$ \\
\hline ESR & NA & 10 & 9 & 2 & 8 & 5 & 6 & 15 & 7 & 9 & 5 & 6 & 15 & $\mathrm{~mm} / \mathrm{h}$ \\
\hline WBC & 6.8 & 6.3 & 5.8 & 4.0 & 2.6 & 2.9 & 3.4 & 3.2 & 2.8 & 2.8 & 3.4 & 3.1 & 3.3 & g/l \\
\hline $\mathrm{RBC}$ & 4.42 & 4.7 & 4.5 & 4.5 & 4.4 & 4.5 & 4.3 & 4.2 & 4.4 & 4.4 & 4.4 & 4.4 & 4.8 & $\mathrm{~T} / \mathrm{l}$ \\
\hline Hemoglobin & 133 & 146 & 140 & 137 & 134 & 135 & 129 & 127 & 136 & 133 & 136 & 135 & 146 & g/l \\
\hline Hematocrit & 0.41 & 0.45 & 0.42 & 0.42 & 0.42 & 0.42 & 0.39 & 0.39 & 0.41 & 0.41 & 0.42 & 0.42 & 0.45 & $1 / 1$ \\
\hline Thrombocyte & 240 & 217 & 211 & 171 & 142 & 166 & 166 & 168 & 171 & 147 & 159 & 159 & 151 & g/l \\
\hline TSH & NA & 1.26 & 1.19 & 1.63 & 1.64 & 1.67 & 1.99 & NA & 1.79 & 1.88 & 1.87 & 2.12 & 2.07 & $\mathrm{mIU} / \mathrm{l}$ \\
\hline CA 19-9 & 7.28 & NA & 7.28 & 7.60 & 8.10 & 7.60 & 7.80 & 7.60 & 6.50 & 3.60 & 6.60 & 12.2 & 12 & $\mathrm{U} / \mathrm{mL}$ \\
\hline CEA & 7.26 & NA & 7.26 & 6.90 & 4.50 & 3.20 & 2.80 & 2.20 & 1.90 & 1.80 & 1.20 & 1.90 & 2.40 & $\mu \mathrm{g} / \mathrm{L}$ \\
\hline urinary ketones & + & +++ & NA & +++ & NA & + & +++ & NA & + & + & + & ++ & NA & \\
\hline
\end{tabular}

Laboratory measurements at diagnosis onset on a standard diet with three medicines (on 15.05.2015) and on the next 12 laboratory exams during follow-up while on the paleolithic ketogenic diet without medication. Following diet onset laboratory parameters normalized. Mildly elevated potassium levels (those above $5.1 \mathrm{mmol} / \mathrm{l}$ ) should not be regarded as pathological given that blood samples were transported. * indicates that the laboratory workup was performed during the radiotherapy period, NA indicates that a given parameter was not measured

Abbreviations: WBC: white blood cell count, RBC: red blood cell count, Hgb: hemoglobin, Hct: hematocrit, CRP: C-reactive protein, GOT: glutamateoxaloacetate transaminase, GPT: glutamate-pyruvate transaminase, GGT: gamma-glutamyl transferase, T. protein: total protein, HbA1c: glycated hemoglobin, T. cholesterol: total cholesterol, HDL: high density lipoprotein, LDL: low density lipoprotein, NA: data not available, LDH: lactate dehydrogenase, ALP: alkaline phosphatase, ESR: erythrocyte sedimentation rate, TSH: thyroid stimulating hormone. 
Table 2.

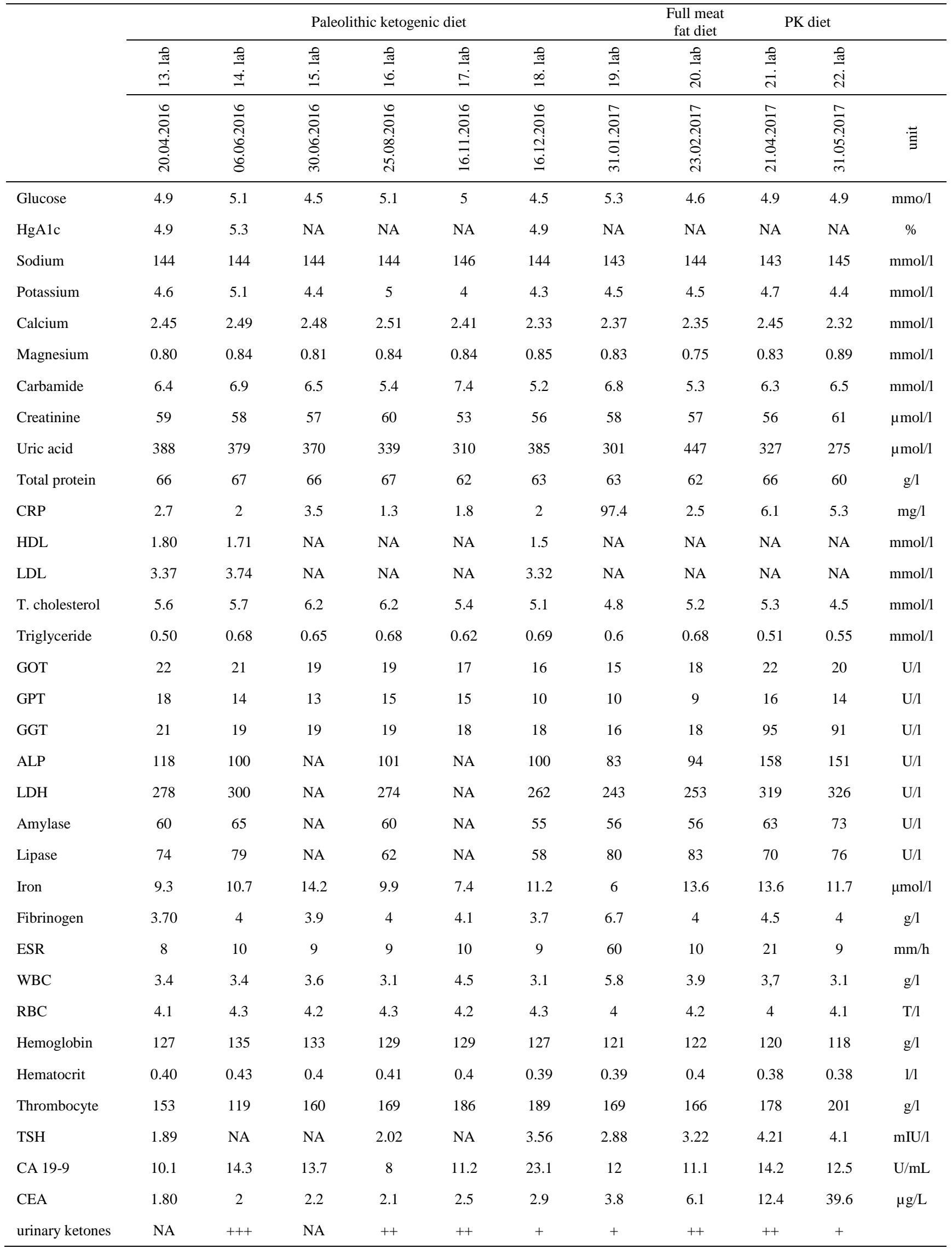

Laboratory measurements between 20.04.2016 and 31.05.2017 on the paleolithic ketogenic diet. Laboratory exam on 31 Jan 2017 showed a marked elevation in all three inflammatory markers (CRP, fibrinogen and ESR) along with decreased iron level. The patient was then started on the strictest version of the paleolithic ketogenic diet, the full meat-fat diet. The laboratory exam, performed at the end of the three weeks period of the full meat-fat diet, showed that all three inflammatory markers markedly decreased and iron levels normalized. Thereafter, along with decreased adherence, laboratory markers deteriorated again. 


\subsection{Laboratory Data}

Laboratory workups were carried out 22 times during follow-up (Table 1 and Table 2) on average, glucose level was $5 \mathrm{mmol} / \mathrm{l}$ (range: 4.3-5.8 mmol/l) while glycated hemoglobin levels averaged 5\% (range: 4.8-5.3\%). Lipase enzyme levels were elevated throughout the entire follow-up while amylase remained normal. Renal and liver function as well as ions including magnesium were normal. Inflammatory markers C-reactive protein, erythrocyte sedimentation rate (ESR) and fibrinogen decreased upon diet onset and remained relatively low on the diet except for the measurement on 31 Jan 2017.

Total cholesterol and LDL cholesterol were slightly elevated while triglyceride remained low. Uric acid elevated following diet onset, then returned to normal within five weeks. His iron level that was low at diet onset (5.6 $\mu \mathrm{mol} / \mathrm{l})$, as it is typical for colorectal cancer. In two weeks after diet onset his iron level increased markedly $(11.4 \mu \mathrm{mol} / \mathrm{l})$. Then first along with radiation therapy and then along with decreased dietary adherence his iron levels were lower. However, laboratory measurement performed after the three weeks on the full meat-fat diet, again showed a marked increase in his iron level. Notably, there was a tendency for inflammatory markers and iron level moving in opposite directions on consecutive measurements (Figure 3).

At the time of diagnosis onset, tumor marker CEA was elevated $(7.26 \mu \mathrm{g} / 1$, normal range: $0-2.5 \mu \mathrm{g} / 1)$ while CA19-9 was in the normal range $(7.28 \mathrm{U} / \mathrm{ml}$, normal range: 0-30.9 $\mathrm{U} / \mathrm{ml}$ ). Following diet onset both tumor markers decreased gradually. Importantly, the decreasing tendency continued beyond the completion of the radiotherapy. Subsequently, in the period of decreased dietary adherence, both tumor markers elevated (Figure 4).

Thyroid function as assessed by TSH (thyroid stimulating hormone) levels was normal during the entire follow-up. FT4 and FT3 levels as measured on 20 Apr 2016 were somewhat lower than the lower limit of the normal range (FT4: 10,99 pmol/l; FT3: 3,45 pmol/L) which can be regarded as normal on a low carbohydrate diet. Vitamin levels as measured on 20 Apr 2016 were normal with vitamin D (25(OH)D) being of 80,9 nmol/l (normal range: $>75 \mathrm{nmol} / \mathrm{l}$ ), folic acid level of $16.4 \mathrm{nmol} / \mathrm{l}$ (normal range: $12.9-54.4 \mathrm{nmol} /$ ) and vitamin B12 level of 221 pmol/l (normal range: 156672 pmol/l). Urinary ketones were positive on each occasion.

\subsection{Ultrasound}

Ultrasound of the abdomen and pelvis was carried out eight times during follow-up and was analysed by the same investigator. This showed a gradual shrinking of the tumor across the first four examinations (Table 3). The fourth examination, on 21 Oct 2015, also indicated a more echo rich pattern of the bowel, a sign indicative of a structural regression. The next ultrasound examination on 05 Apr 2016 (corresponding of the period of decreased dietary adherence) indicated minor progression. The next examination, on 27 Aug 2016, showed relatively stable tumor size while the subsequent exam, on 19 Oct 2016, showed regression. The last exam on 08 March 2017 showed stable tumor size. No enlarged lymph nodes were seen on any ultrasound examination.

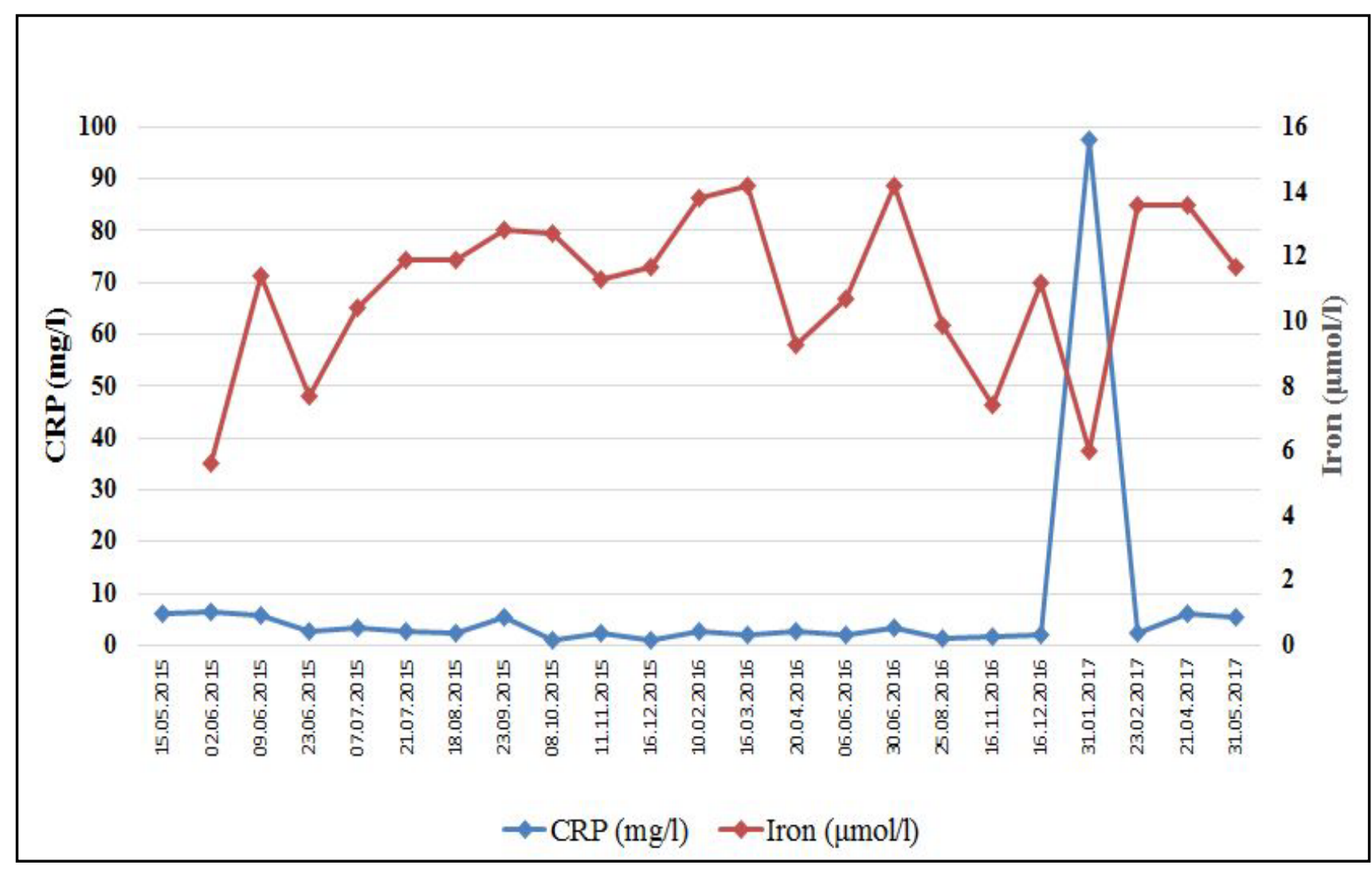

Figure 3. Serum levels of CRP and iron on consecutive laboratory workups. Note the tendency for CRP and iron moving in opposite direction and the normalization of both CRP and iron levels after three weeks on the full meat-fat diet 


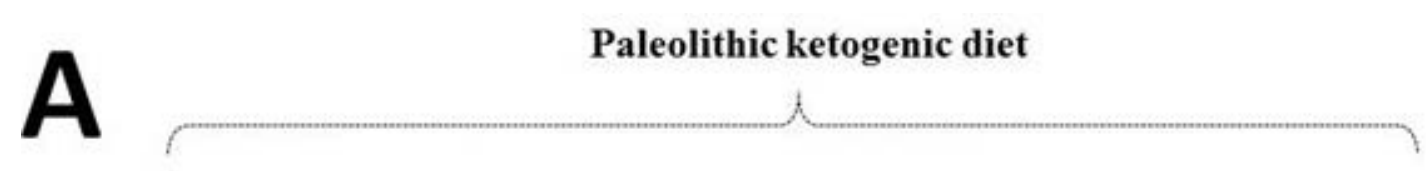

Paleolithic ketogenic diet
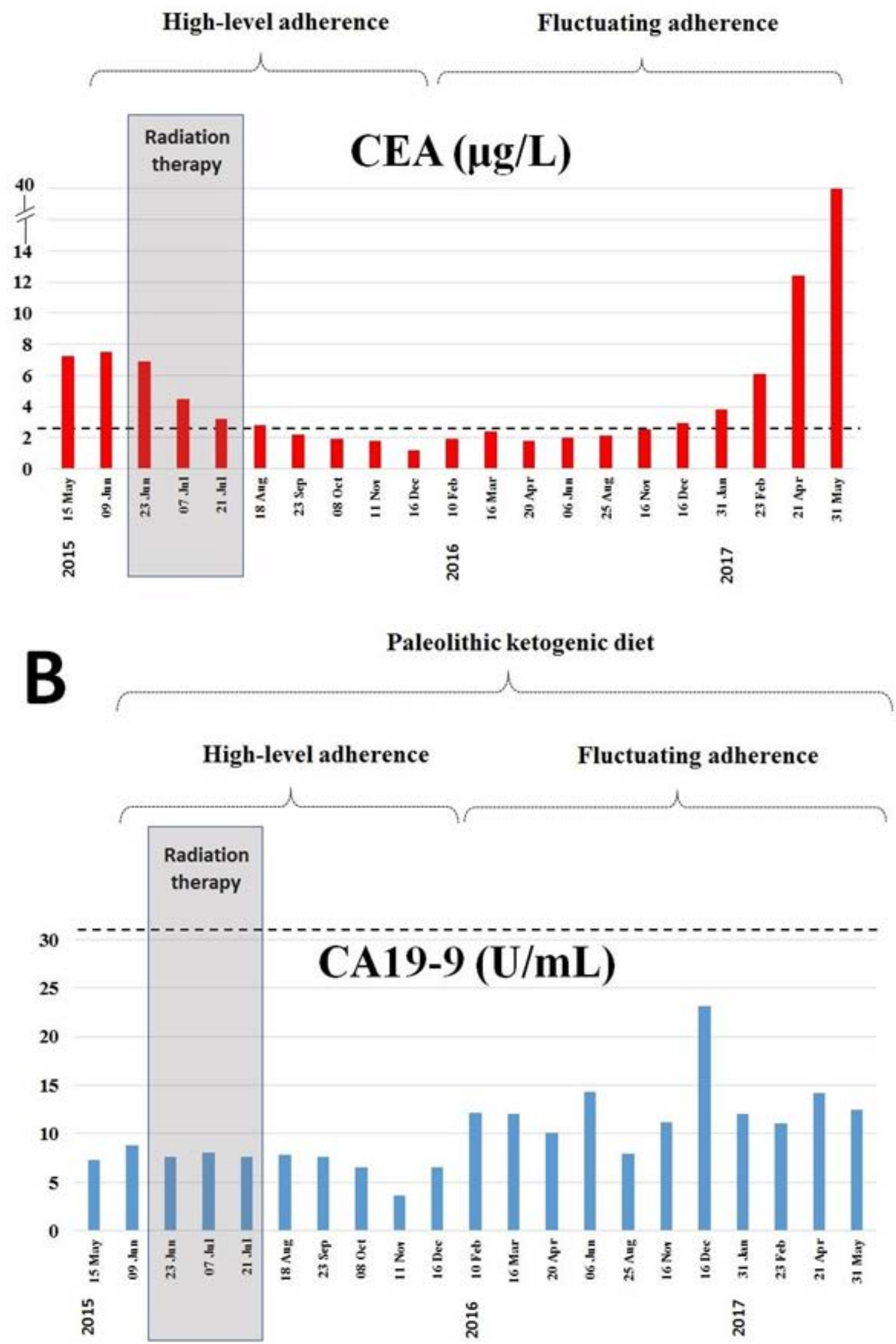

Figure 4. Level of tumor markers CEA and CA 19-9 at diagnosis onset (15 May 2015) on the standard diet and during the 21 months on the paleolithic ketogenic diet. Horizontal dotted line indicates the upper level of the normal range. Grey bars indicate the timing of the radiotherapy. Decreasing tendency of CEA and CA19-9 continued beyond the completion of the radiotherapy. Thereafter along with decreased dietary adherence increased again 
Table 3.

\begin{tabular}{lcccc}
\hline & Lenght $(\mathrm{cm})$ & Thickness of the wall ventrally $(\mathrm{mm})$ & Thickness of the wall dorsally $(\mathrm{mm})$ & Enlarged lymph nodes \\
\hline 06 Jun 2015 & 8.5 & 10.6 & 12 & no \\
23 Jun 2015 & 7 & 10 & 10 & no \\
11 Jul 2015 & 4 & 9.5 & 9.5 & no \\
21 Oct 2016* & 5 & 9 & 10 & no \\
05 Apr 2016 & 7 & 13 & 10 & no \\
27 Aug 2016 & 7 & 15 & 9.5 & no \\
19 Oct 2016 & 5 & 15 & 9.5 & no \\
08 March 2017 & 5 & 15 & $9-12$ & no \\
\hline
\end{tabular}

Lenght of the tumor (thickening of the rectal wall) and thickness of the rectal wall ventrally and dorsally as shown by ultrasound imaging. * indicates that the structure became more echo rich suggesting regression.

Table 4.

\begin{tabular}{ccccc}
\hline & Lenght $(\mathrm{cm})$ & Pathological thickening of the wall $(\mathrm{mm})$ & Diameter of the lumen $(\mathrm{mm})$ & Number of lymph nodes \\
\hline 29 May 2015 & 5.8 & $12-19$ & $17-20$ & $6<$ \\
16 Sep 2015 & $3-3.7$ & $7-9$ & $15-20$ & 0 \\
03 Feb 2016 & $3.2-4$ & $7-12$ & 20 & 2 \\
09 Jun 2016 & $3.2-4$ & $7-15$ & 20 & $2 *$ \\
09 Jan 2017 & $3.2-4$ & $7-12$ & 20 & 2 \\
26 Apr 2017 & $3.2-4$ & $7-12$ & 20 & 2 \\
\hline
\end{tabular}

Length of the tumor, thickening of the wall, lumen diameter and lymph nodes as shown by the MRI rectum imaging. * indicates smaller lymph nodes as compared to the previous exam.

$$
\text { 29.05.2015 }
$$

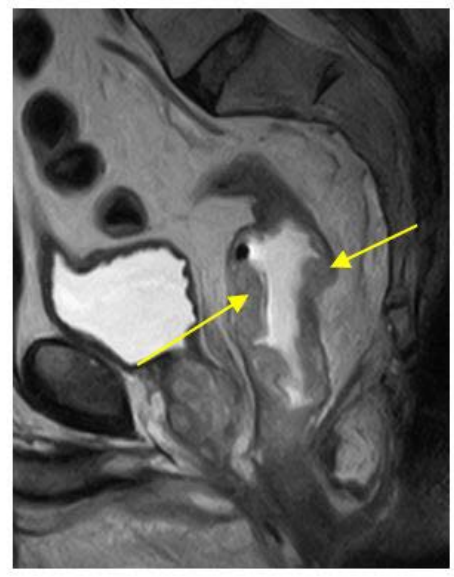

09.06 .2016

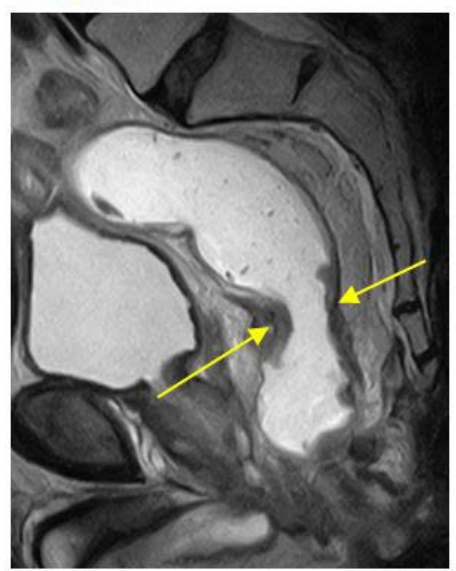

16.09.2015

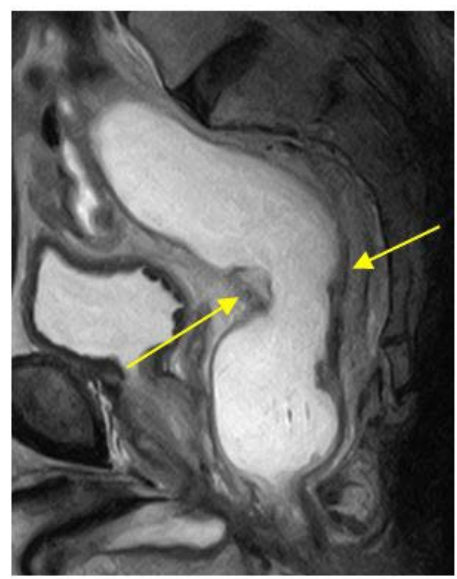

09.01 .2017

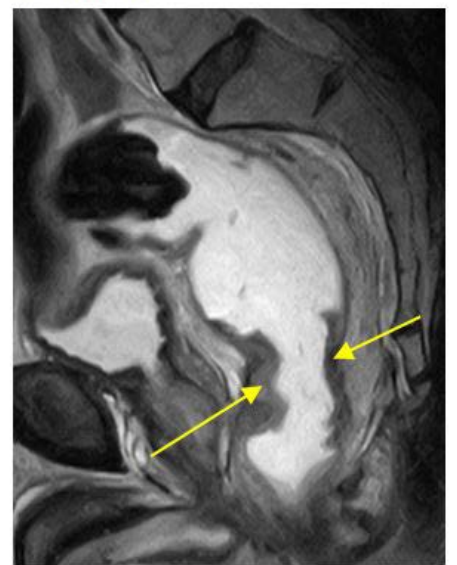

03.02 .2016

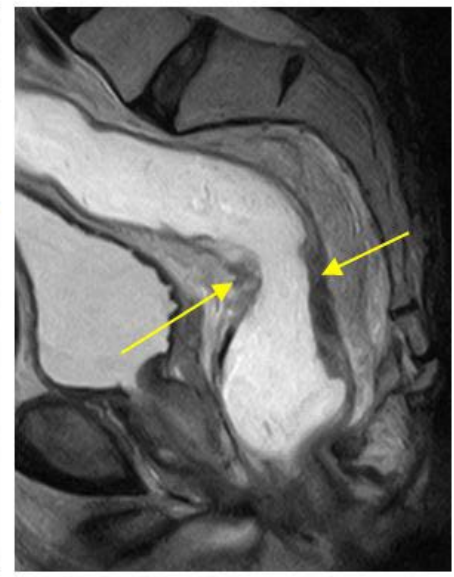

26.04.2017

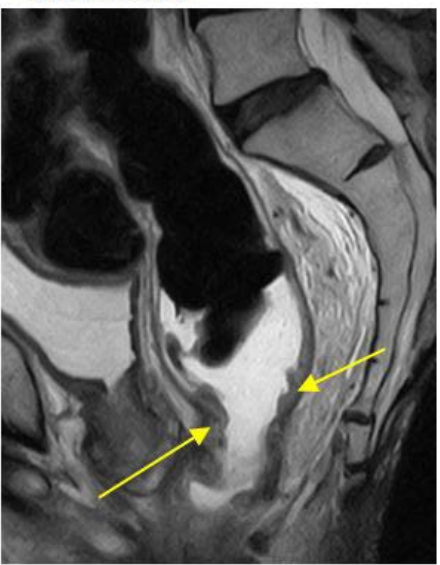

Figure 5. MRI scan showing the rectal tumor at the onset of the paleolithic ketogenic diet (29.05.2015) and on the five follow-up scans. Note the major regression on the first follow-up scan (on 16.09.2015), then the relatively stable tumor size on the next five scans 

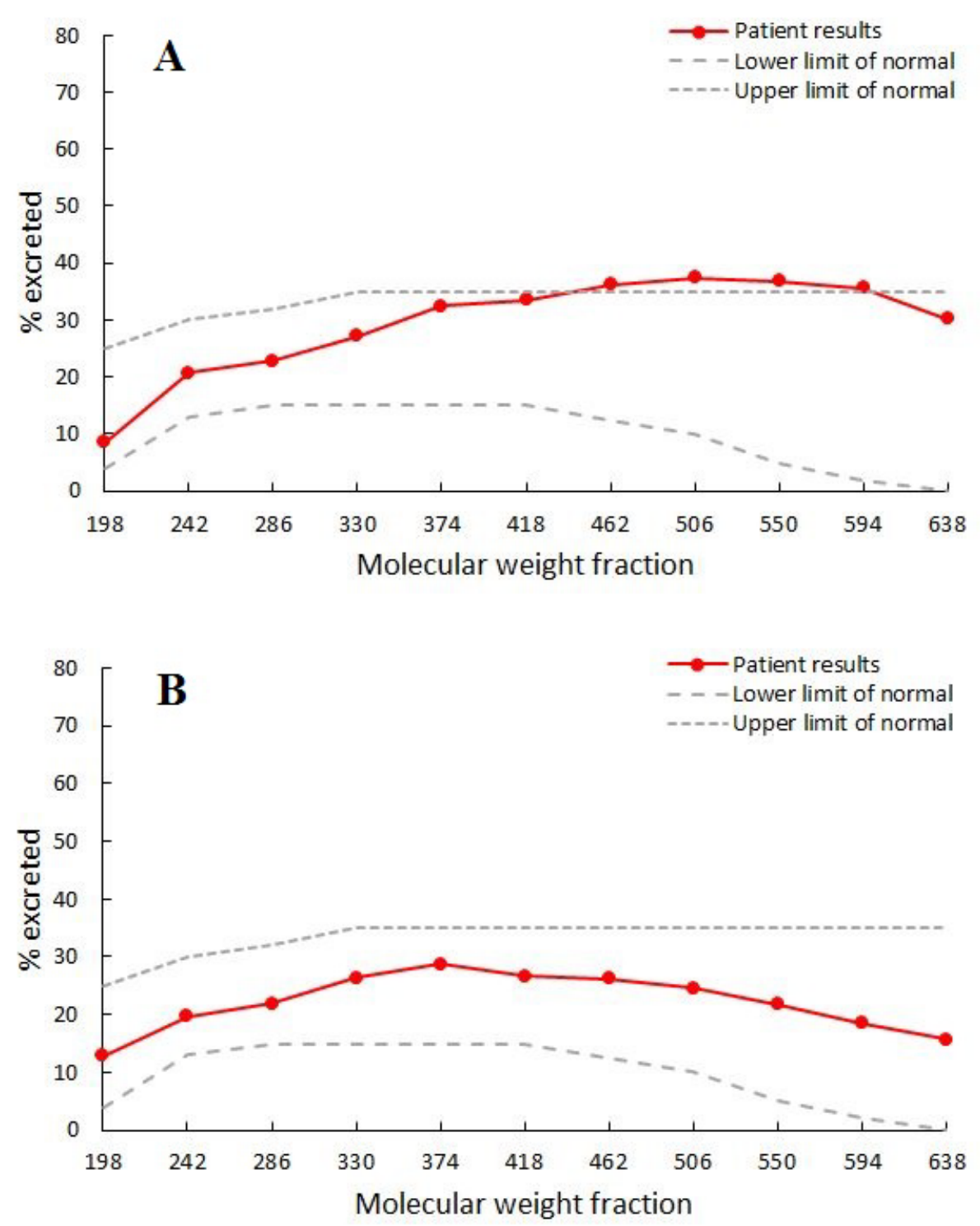

Figure 6. PEG 400 challenge test showing moderate increase in intestinal permeability at 10 months of the diet (A) and normal intestinal permeability at 23 months of the diet (B)

\subsection{MRI of the Pelvis}

Upon the onset of the diet (on 29.05.2015) MRI rectum imaging with ultra sound gel was performed which showed thickening of the bowel wall $5.8 \mathrm{~cm}$ long. The wall was involved circumferentially but did not involve the mesorectal fascia. The first follow-up, on 16 Sep 2015, showed major regression while the second follow-up, on 03 Feb 2016 (associated with decreased dietary adherence), showed minor progression as indicated by the size of the tumor and the number of lymph nodes (Table 4, Figure 5). The next exam (on 09 Jun 2016) showed stable tumor size but a decrease in the size of the lymph nodes. The two last exams (on 09 Jan 2017 and 26 Apr 2017) showed stable tumor size. During the entire follow-up there was a tendency for the tumor moving distally.

\subsection{Intestinal Permeability Test}

Intestinal permeability was assessed using a polyethylene glycol (PEG 400) challenge test based on the method of Chadwick et al. [26]. PEG 400 contains a mixture of inert water-soluble molecules of 11 different sizes that are absorbed independently of dose, but which display decreasing mucosal transport with increasing molecular size. PEG 400 is also nontoxic, not degraded by intestinal bacteria, not metabolized by tissues, and rapidly excreted in urine. After a 3.0-gram oral dose of PEG, the subject makes a six-hour urine collection. The PEG fractions are acetylated with acetic anhydride, using pyridine as a catalyst, and then quantitated by capillary gas-liquid chromatography. The percentage of each fraction of PEG excreted over 6 hours is calculated.

PEG 400 challenge test performed at 10 months on the diet (on 06 Apr 2016) showed slightly increased permeability to PEG between 462 and 594 molecular weight. A second test performed at 23 months on the diet (on 09 May 2017) showed no abnormal intestinal permeability (Figure 6).

\subsection{Condition and Symptoms}

During the first two weeks, the patient was on the paleolithic ketogenic diet without radiation therapy. For this time period the patient reported bloody stool to 
disappear. Following the onset of radiotherapy rectal bleeding returned. During radiotherapy he also experienced an alteration in bowel habits. Apart from this, radiotherapy resulted in relatively weak side effects. Following the completion of the radiotherapy, bloody stool disappeared and bowel habits normalized again within a few weeks. During most of the follow-up the patient experienced no side effects associated with the diet but reported an increased physical and mental fitness. In association with the diet, the patient also reported an unanticipated resolution of other pre-existing symptoms including haemorrhoids and tinea pedis. In January 2017, during the period of decreased dietary adherence, he reported multiple diarrhea a day as a new symptom. Following the return to the full meat-fat diet for three weeks he reported that diarrhea had ceased. During the last two months, again being associated with decreased dietary adherence, the patient reported an increasing frequency of false urges to defecation as a new symptom. Because of the emergence of these symptoms, twenty-four months after diagnosis onset, the patient underwent surgery for rectal tumor and a stoma was formed. During surgery four hepatic metastases were discovered.

\section{Discussion}

Here we present a case where a rectal adenocarcinoma first showed a gradual regression then remained stable in size in a patient on the paleolithic ketogenic diet. Initially, the patient also received radiotherapy for six weeks which may have contributed to the regression of the tumor. However, the radiation dose delivered is typical for neoadjuvant radiation treatment and would usually be escalated in case that a patient declines surgery; still, regression continued beyond the period of radiotherapy where the paleolithic ketogenic diet was used as a standalone therapy. For the first five months there was a regression evidenced by the lowering level of tumor markers and the shrinkage of the tumor. From the sixth months on, although ketosis remained stable, dietary adherence decreased. Stable tumor size across consecutive MRI scans suggested stabilization but surgery at 24 months revealed the emergence of hepatic metastases.

It is assumed that a combination of radiotherapy and chemotherapy followed by surgery, which is the current standard, decreases recurrence rate [27]. It is, however, difficult to find statistical data as to the effectiveness of radiotherapy alone. In a study, radiotherapy alone did not seem to confer benefits in rectal cancer patients with positive resection margins [28].

Low carbohydrate/ketogenic diets have long been suggested to fight cancer [6]. At the same time, apart from an early study in two children [7] no studies are available demonstrating long-term benefits of ketogenic diets. We believe that the lack of long term positive results with the ketogenic diets can be attributed to an unhealthy composition of the diets used in previous clinical studies. Specifically, the fat component in these diets are typically coming from plant oils and/or fat from dairy and may also contain other components which are absent in the paleolithic ketogenic diet. It is known that plant oils, grains and dairy, among their other possible negative effects, may promote inflammation [17]. On the contrary, the paleolithic ketogenic diet, which is based on animal fat, meat and offal, seems to have an anti-inflammatory effect, as also seen in our other patients on the same diet [18-25]. In addition, while the classical ketogenic diet is known to induce nutrient deficiencies [29], the paleolithic ketogenic diet seems to supply optimal amounts of micronutrients as shown by the absence of deficiency symptoms and the normalization of laboratory parameters including iron, magnesium and vitamin levels, and normal function of the liver, kidney and thyroid gland. The patient reported no side effects but improved physical and mental fitness while on the diet.

The relatively mild radiation-induced side effects while on the paleolithic ketogenic diet are consistent with that reported for cancer patients on the classical ketogenic diet [30]. The case of the patient exemplifies that deviations from the diet, even those not affecting ketosis and blood glucose level, may induce negative changes such as elevation of blood pressure, tumor marker and inflammatory marker levels as well as negative changes on imaging and ultimately disease progression. The patient reported that maintaining ketosis had been easy for him. However, maintaining all restrictions of the diet proved to be hard in the long term. In our patient deviation from diet rules resulted in a peak in inflammation associated with iron deficiency anaemia. Importantly, low iron and high CRP are both regarded as negative predictors of colorectal cancer outcome [31,32]. Low iron, in addition, has been suggested to form part of the inflammatory response [33]. On this background, it is of utmost importance that starting the strictest version of the paleolithic ketogenic diet, the full meat-fat diet, resulted in a rapid normalization of both iron and the inflammatory markers as well as that of the clinical symptoms. A subsequent period of decreased dietary adherence resulted in the worsening of laboratory parameters and emergence of clinical symptoms which lead to rectal surgery and the discovery of new metastases. Altogether the paleolithic ketogenic diet which the patient first followed with high adherence and later with lesser adherence allowed for postponing rectal surgery for two years.

We believe that the mechanism of action of the diet can be explained by two main factors. First, given that cancer cells are unable to use ketones but largely depend on glucose for energy, a dietary shift from carbohydrates toward fat put metabolic stress on tumor cells while sparing healthy cells [34]. Second, we put forward that excluding food items humans are not evolutionary adapted to (including all kind of vegetable oils, milk, dairy products, grains, nightshades, legumes and food additives) promote the normalization of the intestinal barrier function. Such a normalization of the intestinal permeability was seen in our previous patient with Crohn's disease and was also paralleled with clinical improvement [24]. Importantly, increased intestinal permeability has been suggested to promote tumorigenesis in colon cancer specifically and in the rest of the body too [35,36]. In our patient testing for intestinal permeability indicated mildly elevated permeability at 10 months on the diet while normal intestinal permeability at 23 months on the diet indicating that the paleolithic ketogenic diet may normalize intestinal permeability. We put forward that the paleolithic ketogenic diet is the 
evolutionary adapted diet in humans. As such it may have the largest potential for boosting regeneration while producing no side effects and nutritional deficiencies. However, deviations from diet, as also in the case of another patient with Crohn's disease [24] may hinder regeneration.

Considering the low efficacy but predictable side effects of chemotherapy and radiotherapy which is the standard care for colorectal cancer [37], we assume that the paleolithic ketogenic diet we used in this patient was without side effects as well as effective as long as the patient strictly followed the diet. The period of high level dietary adherence was associated with normalization of laboratory parameters and regression while deviations from diet rules were associated with worsening laboratory parameters and ultimately progression. We put forward that adopting the paleolithic ketogenic diet may be a viable treatment option for those colorectal cancer patients who are reluctant to accept the standard treatment but are highly motivated in adopting a diet and remaining free of therapy-induced side effects.

\section{Patient Consent}

Written informed consent was obtained from the patient for publication of this case report.

\section{Conflict of Interest}

The authors declare that they have no conflict of interest. The authors of the present study did not receive support from any organization or fund.

\section{References}

[1] Westman EC, Feinman RD, Mavropoulos JC et al. Lowcarbohydrate nutrition and metabolism. Am J Clin Nutr, 2007; 86: 276-84.

[2] Pérez-Guisado J. Arguments In Favor Of Ketogenic Diets. The Internet Journal of Nutrition and Wellness, 2007; 4: 2.

[3] Kossoff EH. More fat and fewer seizures: dietary therapies for epilepsy. Lancet Neurol, 2004; 3: 415-20.

[4] Ely JTA. Glycemic modulation of tumor tolerance. J Orthomolecular Med, 1996; 11: 23-34.

[5] Klement RJ, Kämmerer U. Is there a role for carbohydrate restriction in the treatment and prevention of cancer? Nutr Metab (Lond), 2011; 8: 75.

[6] Seyfried TN. Cancer as a Metabolic Disease: On the Origin, Management, and Prevention of Cancer. Hoboken, New Jersey, Wiley 2012.

[7] Nebeling LC, Miraldi F, Shurin SB et al. Effects of a ketogenic diet on tumor metabolism and nutritional status in pediatric oncology patients: two case reports. J Am Coll Nutr, 1995; 14: 202-8.

[8] Zuccoli G, Marcello N, Pisanello A et al. Metabolic management of glioblastoma multiforme using standard therapy together with a restricted ketogenic diet: Case Report. Nutr Metab (Lond), 2010; 22, 7: 33.

[9] Schmidt M, Pfetzer N, Schwab M et al. Effects of a ketogenic diet on the quality of life in 16 patients with advanced cancer: A pilot trial. Nutr Metab (Lond), 2011; 8: 54

[10] Fine EJ, Segal-Isaacson CJ, Feinman RD et al. Targeting insulin inhibition as a metabolic therapy in advanced cancer: a pilot safety and feasibility dietary trial in 10 patients. Nutrition, 2010; 28: 1028-35.
[11] Klement RJ, Sweeney RA. Impact of a ketogenic diet intervention during radiotherapy on body composition: I. Initial clinical experience with six prospectively studied patients. BMC Res Notes, 2016; 9: 143.

[12] Rieger J, Bähr O, Maurer GD et al. ERGO: a pilot study of ketogenic diet in recurrent glioblastoma. Int J Oncol, 2014; 44: 1843-52.

[13] Champ CE, Palmer JD, Volek JS et al. Targeting metabolism with a ketogenic diet during the treatment of glioblastoma multiforme. J Neurooncol, 2014; 117: 125-31.

[14] Fine EJ, Segal-Isaacson CJ, Feinman RD et al. Carbohydrate restriction in patients with advanced cancer: a protocol to assess safety and feasibility with an accompanying hypothesis. Community Oncology January 2008.

[15] Manheimer EW, van Zuuren EJ, Fedorowicz Z, Pijl H. Paleolithic nutrition for metabolic syndrome: systematic review and metaanalysis. Am J Clin Nutr, 2015; 102: 922-32.

[16] Voegtlin WL. The stone age diet: based on in-depth studies of human ecology and the diet of man. New York: Vantage Press; 1975.

[17] Cordain L. The paleo diet: lose weight and get healthy by eating the food you were designed to eat. New York: Wiley; 2002.

[18] Tóth C, Clemens Z. Type 1 diabetes mellitus successfully managed with the paleolithic ketogenic diet. Int J Case Rep Images, 2014; 5: 699-703.

[19] Tóth C, Clemens Z. A child with type 1 diabetes mellitus (T1DM) successfully treated with the Paleolithic ketogenic diet: A 19-month insulin freedom. Int J Case Rep Images, 2015; 6: 752-757.

[20] Tóth C, Clemens Z. Successful treatment of a patient with obesity, type 2 diabetes and hypertension with the paleolithic ketogenic diet. Int J Case Rep Images, 2015; 6: 161-167.

[21] Clemens Z, Kelemen A, Fogarasi A. et al. Childhood absence epilepsy successfully treated with the paleolithic ketogenic diet. Neurol Ther, 2013; 2: 71-6.

[22] Clemens, Z, Kelemen, A, Tóth, C. NREM-sleep Associated Epileptiform Discharges Disappeared Following a Shift toward the Paleolithic Ketogenic Diet in a Child with Extensive Cortical Malformation. Am J Med Case Rep, 2015; 3: 212-215.

[23] Tóth C, Clemens Z. Gilbert's syndrome successfully treated with the paleolithic ketogenic diet. Am J Med Case Rep, 2015; 3: 117-120.

[24] Tóth C, Dabóczi A, Howard M et al. Crohn’s disease successfully treated with the paleolithic ketogenic diet. Int J Case Rep Images, 2016; 7: 570-578.

[25] Tóth C, Clemens Z. Halted progression of soft palate cancer in a patient treated with the paleolithic ketogenic diet alone: A 20-months Follow-up. Am J Med Case Rep, 2016; 4: 288-292.

[26] Chadwick VS, Phillips SF, Hofmann AF. Measurements of intestinal permeability using low molecular weight polyethylene glycols (PEG 400). I. Chemical analysis and biological properties of PEG 400. Gastroenterology, 1977; 73: 241-6.

[27] Glimelius B. Neo-adjuvant radiotherapy in rectal cancer. World J Gastroenterol, 2013; 19: 8489-8501.

[28] Marijnen CA, Nagtegaal ID, Kapiteijn E et al. Cooperative investigators of the Dutch Colerectal Cancer Group. Radiotherapy does not compensate for positive resection margins in rectal cancer patients: report of a multicenter randomized trial. Int J Radiat Oncol Biol Phys, 2003; 55: 1311-20.

[29] Kossoff EH. International consensus statement on clinical implementation of the ketogenic diet: agreement, flexibility, and controversy. Epilepsia, 2008; 49 Suppl 8: 11-3.

[30] Seyfried TN. Case Studies and Personal Experiences in Using the Ketogenic Diet for Cancer Management, in Cancer as a Metabolic Disease: On the Origin, Management and Prevention of Cancer, John Wiley \& Sons, Inc., Hoboken, NJ, USA. 2012.

[31] Wessling-Resnick M. Iron Homeostasis and the Inflammatory Response. Annual review of nutrition. 2010; 30: 105-122.

[32] Toiyama Y, Inoue Y, Saigusa S, Kawamura M, Kawamoto A, Okugawa Y, Hiro J, Tanaka K, Mohri Y, Kusunoki M. C-reactive protein as predictor of recurrence in patients with rectal cancer undergoing chemoradiotherapy followed by surgery. Anticancer Res. 2013; Nov; 33(11): 5065-74.

[33] Hamilton W, Lancashire R, Sharp D, Peters TJ, Cheng KK, Marshall T. The importance of anaemia in diagnosing colorectal cancer: a case-control study using electronic primary care records. British Journal of Cancer. 2008; 98(2): 323-327. 
[34] Warburg O. "On the Origin of Cancer Cells". Science, 1956; 123 : 309-14.

[35] Soler AP, Miller RD, Laughlin KV et al. Increased tight junctional permeability is associated with the development of colon cancer Carcinogenesis, 1999; 20: 1425-31.
[36] Lin JE, Snook AE, Li P et al. GUCY2C Opposes Systemic Genotoxic Tumorigenesis by Regulating AKT-Dependent Intestinal Barrier Integrity. PLoS ONE, 2012; 7: 2, e31686.

[37] SEER Stat Fact Sheets: Colon and Rectum Cancer. http://seer.cancer.gov/statfacts/html/colorect.html (Retrieved 13 June 2016) 